\title{
Secure Relay Beamforming in Non-Regenerative Two-Way Relay Cognitive Radio Networks with Energy Harvesting Constraints
}

\author{
Feng-Chuang Xing, Sai Zhao, Gao-Fei Huang, Ming-Xia Lv, Gui-Yun Liu, Guo-Qi Wang, Dong Tang \\ School of Mechanical and Electrical Engineering, Guangzhou University, Guangzhou, Guangdong Province, China \\ E-mail: xingfengchuang@e.gzhu.edu.cn, zhaosai@gzhu.edu.cn, huanggaofei@gzhu.edu.cn, lvmingxia@gzhu.edu.cn, \\ liuguiyun@gzhu.edu.cn, wangguoqi@e.gzhu.edu.cn, tangdong@gzhu.edu.cn
}

\begin{abstract}
-in this paper, we consider the problem of nonregenerative two-way secure relay beamforming (SRB) for cognitive radio network (CRN) under the background of the simultaneous wireless information and power transfer (SWIPT). Our objective is to maximize sum rate under the transmit power constraint at the relay, energy harvesting (EH) constraint at the $\mathrm{EH}$ device and the interference constraint from secondary user (SU). The formulated problem is a nonconvex problem. We firstly propose a two-dimensional (2D) search scheme which provides an upper bound to the formulated problem; for decreasing the computation complexity, we propose a sequential parametric convex approximation (SPCA) based iteration scheme and obtain a local optimal solution. Simulation results have shown that SPCA based iteration scheme performs almost close to 2D search schemes, and has lower computation complexity.
\end{abstract}

Keywords-EH; two-way relay network; CRN; convex optimization

\section{INTRODUCTION}

The need for the efficient use of the scarce spectrum in wireless applications has led to significant interest in the study of cognitive radio system. One possible scheme for the operation of the cognitive radio network is to allow the secondary users to transmit concurrently on the same frequency band as the primary users when the resulting interference power at the receivers is kept below the interference temperature limit [1]. The problem of secure transmission in the presence of an eavesdropper was first studied from an information-theoretic perspective in [2] where Wyner considered a wiretap channel model. Simultaneous wireless information and power transfer, which belongs to energy harvesting $(\mathrm{EH})$ techniques, is a promising approach to solving the energy scarcity problem in energy-constrained wireless network. Motivated by the benefits of multi-antennas techniques, SWIPT for multipleinput-multiple-output (MIMO) and multiple-input-singleoutput (MISO) broadcast channels was investigated in [3][4]. The SWIPT schemes for two-way relay OFDM were studied in [5]. However, to the best of our knowledge, the research on secure relay beamforming in non-regenerative two-way relay cognitive radio networks is missing.

In this paper, the eavesdropper's CSI is perfect at the relay and the sources. In order to solve our formulated problem, we firstly propose a two-dimensional (2D) search scheme; however the computation complexity is high.
Motivated by decreasing the computation complexity, we propose a sequential parametric convex approximation (SPCA) based iteration scheme.

The organization of the paper is as follows. In the Section II, we present the system model of the two-way wiretap channel with one two-way relay node and one eavesdropper. In the Section III, two different schemes, 2D search scheme and SPCA based iteration schemes are investigated, respectively. In Section IV, simulations are taken to illustrate the performance of the two schemes. In Section V, we show the computational complexity of the two schemes that we propose, and finally, in Section VI conclude the paper.

Boldface lowercase and uppercase letters denote vectors and matrices, respectively. The conjugate, transpose, conjugate transpose, Frobenius norm and trace of the matrix $\mathbf{A}$ denoted as $\mathbf{A}^{*}, \mathbf{A}^{\mathrm{T}}, \mathbf{A}^{\mathrm{H}},\|\mathbf{A}\|$, and $\operatorname{tr}(\mathbf{A})$, respectively. The $\otimes$ denotes Kronecker product. $\operatorname{vec}(\mathbf{A})$ denotes to stack the columns of a matrix $\mathbf{A}$ into a single vector $\mathbf{a} . \operatorname{Re}(\mathbf{A})$ denotes the real part of a matrix of $\mathbf{A}$. $\|\mathbf{a}\|$ denotes the Euclidean norm of vector $\mathbf{a} . \lambda_{\max }(\mathbf{A})$ and $\lambda_{\text {min }}(\mathbf{A})$ denote the maximum and minimum eigenvalues of $\mathbf{A}$, respectively. By $\mathbf{A} \geq 0$ or $\mathbf{A} \succ 0$, we mean that the matrix $\mathbf{A}$ is positive semidefinite or positive definite respectively.

\section{SYSTEM MODEL}

In this paper, we consider a dual-hop cognitive channel with two users source1 and source2, a primary user, an eavesdropper, an EH device and a relay. The nonregenerative relay typically adopts amplify-and-forward (AF) protocol. The two-way relay, equipped with Nantennas, is responsible to establish reliable and secure communications between source1 and source2, to transfer power to the EH receiver. Every node is equipped with single antenna except the relay. As shown in Fig. 1.

All the terminals and two-way relay node are in halfduplex mode. We assume that there is no direct link between two sources and all the channels remain unchanged during one slot. Thus, the quasi-static channels are considered in this paper. 


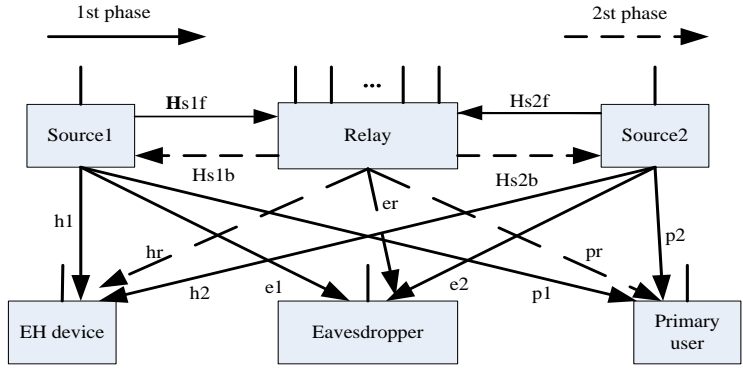

Figure 1. The system model of two-way relay cognitive radio network with eavesdropper and $\mathrm{EH}$ device

The source1 and the source 2 exchange the information symbols through the relay are divide into two phases. In the first phase, Source1 and Source2, at the same time, transmit the symbols $x_{1}$ and $x_{2}$ to the two-way relay, eavesdropper, EH device and PU, respectively. $\mathbf{y}_{\mathbf{R}}$ stands for the relay received signal

$$
\mathbf{y}_{\mathbf{R}}=\mathbf{H}_{\mathrm{s} 1 \mathrm{f}} x_{1}+\mathbf{H}_{\mathbf{s} \mathbf{f f}} x_{2}+\mathbf{n}_{\mathbf{R}}(1)
$$

where $\mathbf{H}_{s 1 f} \in C^{N \times 1}$ denotes channel response from the Source1 to the relay, $\mathbf{H}_{s 2 f} \in C^{N \times 1}$ denotes channel response from the source2 to the relay, $\mathbf{n}_{R} \in C^{N \times 1}$ is the additive Gaussian noise (AWGN) vector at the relay, which has zero mean and covariance matrix $\sigma^{2} \mathbf{I} \cdot y_{E, 1}$ stands for the signal by the eavesdropper received

$$
y_{E, 1}=e_{1} x_{1}+e_{2} x_{2}+n_{E, 1}
$$

$e_{1}$ denotes channel response from the source1 to the Eavesdropper, $e_{2}$ denotes channel response from the source 2 to the eavesdropper, $n_{E, 1}$ is the AWGN at the eavesdropper, which has zero mean and variance $\sigma^{2} \cdot y_{p, 1}$ and $\mathrm{E}_{p 1}$ stands for the signal and energy, respectively, received by the $\mathrm{PU}$

$$
\begin{gathered}
y_{P, 1}=p_{1} x_{1}+p_{2} x_{2}+n_{p, 1} \\
\mathrm{E}_{p 1}=\mathrm{E}\left(P_{1} p_{1}^{2}+P_{2} p_{2}^{2}\right)
\end{gathered}
$$

$p_{1}$ denotes channel response from the sourcel to the PU. $\mathrm{P}_{1}=E\left\lfloor\left|x_{1}\right|^{2}\right\rfloor$, which stands for the source1 average transmitted signal energy. $p_{2}$ denotes channel response from the source2 to the PU. $\left.\mathrm{P}_{2}=\left.E|| x_{2}\right|^{2}\right]$, which stands for the source 2 average transmitted signal energy. $n_{p, 1}$ is the AWGN at the PU, which has zero mean and variance $\sigma^{2}$.

The energy received by EH device can express as

$$
\mathrm{E}_{1}=\rho\left(\mathrm{P}_{1}\left|h_{1}\right|^{2}+\mathrm{P}_{2}\left|h_{2}\right|^{2}\right)
$$

where $h_{1}$ denotes channel response from the source1 to the the EH device. The factor $\rho$ denotes the efficiency of EH device, we assume that the EH device efficiency $\rho=1$.

In the second phase, the relay needs beamforming matrix to enlarge the received signal, and then forwards the enlarged signal to two sources. We utilize $\mathbf{W} \in \mathbf{C}^{\mathbf{N} \times \mathbf{N}}$ as beamforming matrix. The transmitted signal from relay can be expressed as

$$
\mathbf{Y}_{\mathbf{R}}=\mathbf{W y}_{\mathbf{R}}
$$

The minimum transmit energy of relay can be expressed as [6]

$$
\mathrm{E}\left[\left.\mathbf{Y}_{\mathrm{R}}\right|^{2}\right]=\mathrm{E}\left\{\operatorname{tr}\left(\mathbf{W} \cdot \mathbf{y}_{R} \cdot \mathbf{y}_{R}^{H} \cdot \mathbf{W}\right)\right\}=\operatorname{tr}\left(\mathbf{W} \cdot \mathbf{Y}_{y} \cdot \mathbf{W}^{H}\right)(7)
$$

where $\mathbf{Y}_{y}=\mathbf{y}_{R} \cdot \mathbf{y}_{R}^{H}$.

By using the equality (8) and (9)

$$
\begin{aligned}
\operatorname{tr}\left(\mathbf{A}^{H} \mathbf{B}\right) & =\operatorname{vec}(\mathbf{A})^{H} \operatorname{vec}(\mathbf{B}) \\
\operatorname{vec}(\mathbf{A B C}) & =\left(\mathbf{C}^{T} \otimes \mathbf{A}\right) \operatorname{vec}(\mathbf{B})
\end{aligned}
$$

The minimum transmit energy of relay can be transformed as

$$
\left.\mathrm{E}\left\|\mathbf{Y}_{R}\right\|^{2}\right]=\mathbf{w}^{H}\left(\mathbf{Y}_{y}^{T} \otimes \mathbf{I}\right) \mathbf{w}
$$

where $\mathbf{w}=\operatorname{vec}(\mathbf{W})$.

The received signal at source1 and source 2 can be expressed as

$$
\begin{aligned}
& y_{s 1}=\mathbf{H}_{S 1 b}^{T} \mathbf{W H}_{\mathrm{S} 1 f} x_{1}+\mathbf{H}_{S 1 b}^{T} \mathbf{W H}_{\mathrm{S} 2 f} x_{2}+\mathbf{H}_{S 1 b}^{T} \mathbf{W} \mathbf{n}_{R} \\
& +n_{S 1} \\
& y_{s 2}=\mathbf{H}_{S 2 b}^{T} \mathbf{W H}_{\mathrm{S} 1 f} x_{1}+\mathbf{H}_{S 2 b}^{T} \mathbf{W} \mathbf{H}_{\mathrm{S} 2 f} x_{2}+\mathbf{H}_{S 2 b}^{T} \mathbf{W} \mathbf{n}_{R} \\
& +n_{S 2}
\end{aligned}
$$

where $\mathbf{H}_{S 1 b} \in C^{N \times 1}$ denotes channel response from the relay to source1. $n_{S 1}$ is a AWGN at Source1, which has zero mean and variance $\sigma^{2} . \mathbf{H}_{S 2 b} \in C^{N \times 1}$ denotes channel 
response from the relay to the source $2, n_{S 2}$ is a AWGN at Source2, which has zero mean and variance $\sigma^{2}$.

The received signal at Eavesdropper can be expressed as

$$
y_{E, 2}=\mathbf{e}_{r}^{T} \mathbf{W H} \mathbf{H}_{S 1 f} x_{1}+\mathbf{e}_{r}^{T} \mathbf{W} \mathbf{H}_{S 2 f} x_{21}+\mathbf{e}_{r}^{T} \mathbf{W} \mathbf{n}_{R}+n_{E, 2}
$$

where $\mathbf{e}_{r} \in C^{N \times 1}$ denotes channel response from the relay to the Eavesdropper. $n_{E, 2}$ is a AWGN at Eavesdropper, which has zero mean and variance $\sigma^{2}$.

The received signal and energy at PU can be expressed as

$$
\begin{gathered}
y_{p, 2}=\mathbf{p}_{r}^{T} \mathbf{W} \mathbf{H}_{S 1 f} x_{1}+\mathbf{p}_{r}^{T} \mathbf{W} \mathbf{H}_{S 2 f} x_{2}+\mathbf{p}_{r}^{T} \mathbf{W} \mathbf{n}_{R}+n_{p, 2} \\
E_{p 2}=\mathbf{W}^{H} \mathbf{A}_{P U} \mathbf{W}
\end{gathered}
$$

where $\mathbf{A}_{P U}=\left(P_{1} \mathbf{H}_{S 1 f} \mathbf{H}_{S 1 f}^{H}+P_{2} \mathbf{H}_{S 2 f} \mathbf{H}_{S 2 f}^{H}+\sigma^{2} \mathbf{I}\right)^{T} \otimes \mathbf{p}_{r}^{*} \mathbf{p}_{r}^{T}$. $\mathbf{p}_{r} \in C^{N \times 1}$ denotes channel response from the relay to the PU and $n_{p, 2}$ is a AWGN at PU, which has zero mean and variance $\sigma^{2}$.

The first term in (11) and the second term in (12) is the backward self-interference. We assume that each terminal knows both the channels associated itself with the relay nodes and the weighted coefficient matrix $\mathbf{W}$. It can subtract the backward self-interference from itself and only obtain the desired information from the other one. After this operation, we can get (16) and (17)

$$
\begin{aligned}
& y_{s 1}=\mathbf{H}_{S 1 b}^{T} \mathbf{W H}_{\mathrm{s} 2 f} x_{2}+\mathbf{H}_{S 1 b}^{T} \mathbf{W n}_{R}+n_{S 1} \\
& y_{s 2}=\mathbf{H}_{S 2 b}^{T} \mathbf{W H}_{\mathrm{S} 1 f} x_{1}+\mathbf{H}_{S 2 b}^{T} \mathbf{W n}_{R}+n_{S 2}
\end{aligned}
$$

From (15) and (16), the received signal-to-noise ratio (SNR) at Source1 and Source 2 are $\gamma_{1}$ and $\gamma_{2}$

$$
\begin{aligned}
\gamma_{1} & =\frac{\mathbf{P}_{2} \mathbf{w}\left(\mathbf{H}_{S 2 f}^{H} \mathbf{H}_{S 2 f} \otimes \mathbf{H}_{S 1 b}^{T} \mathbf{H}_{S 1 b}^{*}\right) \mathbf{w}}{\mathbf{w}^{H}\left(\sigma^{2} \mathbf{I} \otimes \mathbf{H}_{S 1 b}^{T} \mathbf{H}_{S 1 b}^{*}\right) \mathbf{w}+\sigma^{2}} \\
\gamma_{2} & =\frac{\mathbf{P}_{1} \mathbf{w}^{\mathrm{H}}\left(\mathbf{H}_{S 1 f}^{H} \mathbf{H}_{S 1 f} \otimes \mathbf{H}_{S 2 b}^{T} \mathbf{H}_{S 2 b}^{*}\right) \mathbf{w}}{\mathbf{w}^{H}\left(\sigma^{2} \mathbf{I} \otimes \mathbf{H}_{S 2 b}^{T} \mathbf{H}_{S 2 b}^{*}\right) \mathbf{w}+\sigma^{2}}
\end{aligned}
$$

In the second phase, the harvest energy of EH device can be expressed as

$$
\mathrm{E}_{2}=\mathbf{w}^{H}\left(\mathbf{A}_{E H 2}\right) \mathbf{w}
$$

where $\mathbf{A}_{\mathrm{EH} 2}=\left(\mathrm{P}_{1} \mathbf{H}_{\mathrm{S} 1 f} \mathbf{H}_{S 1 f}^{H}+\mathrm{P}_{2} \mathbf{H}_{\mathrm{S} 2 f} \mathbf{H}_{S 2 f}^{H}+\sigma^{2} \mathbf{I}\right)^{T} \otimes \mathbf{h}_{r}^{*} \mathbf{h}_{r}^{T}$ $\mathbf{h}_{r} \in C^{N \times 1}$ denotes the channel response vector from relay to $\mathrm{EH}$ device. In both phases, the eavesdropper has chance to receive the information which has been transmitted from source 1 , source 2 and two-way relay. The information can be expressed as [7]

$$
\mathbf{y}_{e}=\mathbf{H}_{e} \mathbf{x}+\mathbf{n}_{e}
$$

where $\mathbf{y}_{e}=\left[y_{E, 1}, y_{E, 2}\right]^{\mathrm{T}}$

$$
\begin{gathered}
\mathbf{H}_{e}=\left[\begin{array}{cc}
e_{1} & e_{2} \\
\mathbf{e}_{r}^{T} \mathbf{W H}_{S 1 f} & \mathbf{e}_{r}^{T} \mathbf{W H}_{S 2 f}
\end{array}\right], \mathbf{x}=\left[\begin{array}{ll}
x_{1} & x_{2}
\end{array}\right]^{T} \\
\mathbf{n}_{e}=\left[\begin{array}{c}
n_{E, 1} \\
\mathbf{e}_{r}^{T} \mathbf{W n}_{R}+n_{E, 2}
\end{array}\right]
\end{gathered}
$$

We employ matrices to describe the information of the Eavesdropper. The capacity of the eavesdropper got information can denote as (22) in both phases

$$
\mathbf{R}_{e}=\frac{1}{2} \log _{2} \operatorname{det}\left(\mathbf{I}+\mathbf{H}_{e} P \mathbf{H}_{e}^{H} \mathbf{R}_{\mathrm{E}}^{-1}\right)
$$

Where

$\mathbf{R}_{E}=\operatorname{diag}\left(\sigma^{2}, \sigma^{2}\left(1+\mathbf{w}^{\mathrm{H}} R_{c c} \mathbf{w}\right)\right), \mathbf{P}=\operatorname{diag}\left(P_{1}, P_{2}\right)$.

For (22), we can denote

$$
\mathbf{R}_{e}=\frac{1}{2} \log _{2} \frac{\mathbf{w}^{H} \mathbf{Q}_{\mathbf{e}} \mathbf{w}+\alpha}{\sigma^{4}\left(1+\mathbf{w}^{H} \mathbf{R}_{c c} \mathbf{w}\right)}
$$

$$
\mathbf{Q}_{e}=\left(P_{1} P_{2}\left|e_{2}\right|^{2}+P_{1} \sigma^{2}\right) \mathbf{R}_{c f}+\left(P_{1} P_{2}\left|e_{1}\right|^{2}+P_{2} \sigma^{2}\right) \mathbf{R}_{c g}
$$$$
+\alpha \mathbf{R}_{c c}-P_{1} P_{2} e_{1} e_{2}^{*} \mathbf{a}_{c f}^{*} \mathbf{a}_{c g}^{T}-P_{1} P_{2} e_{2} e_{1}^{*} \mathbf{a}_{c g}^{*} \mathbf{a}_{c f}^{T}
$$$$
\mathbf{R}_{c f}=\mathbf{a}_{c f} \mathbf{a}_{c f}^{H}, \mathbf{R}_{c g}=\mathbf{a}_{c g} \mathbf{a}_{c g}^{H}, \mathbf{R}_{c c}=\left[\mathbf{I} \otimes\left(\mathbf{e}_{r} \mathbf{e}_{r}^{\mathrm{H}}\right)\right]
$$$$
\mathbf{a}_{c f}=\operatorname{vec}\left(e_{r} \mathbf{H}_{S 1 f}^{T}\right), \mathbf{a}_{c g}=\operatorname{vec}\left(e_{r} \mathbf{H}_{S 2 f}^{T}\right),
$$$$
\alpha=\mathrm{P}_{1} \sigma^{2}\left|e_{1}\right|^{2}+\mathrm{P}_{2} \sigma^{2}\left|e_{2}\right|^{2}+\sigma^{4} \text {. }
$$

The sum rate of two-way relay cognitive networks can be expressed as

$$
\mathbf{R}_{S}=\left[\frac{1}{2} \log _{2}\left(1+\gamma_{1}\right)+\frac{1}{2} \log _{2}\left(1+\gamma_{2}\right)-R_{e}\right]^{+}
$$

where $[a]^{+}=\max (0, a)$.

The constraint of the transmit power at relay can be 
expressed as

$$
\mathbf{w}^{\mathrm{H}}\left(\mathbf{Y}_{y}^{T} \otimes \mathbf{I}\right) \mathbf{w} \leq P_{t h}
$$

Since considering the normal working conditions of the relay equipment, we assume that the $\mathrm{P}_{t h}$ denotes the transmit power threshold at the relay.

The constraint of the EH device can be expressed as

$$
E_{1}+E_{2} \geq Q_{t h}
$$

Since considering the normal working conditions of the energy harvesting equipment, we assume that the $Q_{t h}$ denotes the energy harvesting threshold at the $\mathrm{EH}$ device.

The constraint of the interference temperature at PU can be expressed as

$$
E_{p 1}+E_{p 2} \leq I_{t h}
$$

In this paper, we employ the underlay model of cognitive radio networks, namely, the interference of second user to the PU should lower than a threshold value $I_{t h}$.

According to [8] and [9], $Q_{t h}$ should be chosen such that $\left[0, Q_{\max }\right]$, where $Q_{\max }$ can be expressed as

$$
\mathrm{Q}_{\max }=\mathrm{E}_{1}+\max \left(E_{2}\right)=\mathrm{E}_{1}+\lambda_{\max }\left(\mathbf{A}_{E H_{2}}\right)
$$

Since the $I_{\text {th }}$ is similar to $Q_{\max }$, using the same approach, we set the $I_{t h}$. It should be chosen such that $\left[0, \mathrm{I}_{\max }\right]$, where $\mathrm{I}_{\max }$ can be expressed as

$$
\mathrm{I}_{\text {max }}=\mathrm{E}_{p 1}+\max \left(E_{p 2}\right)=\mathrm{E}_{p 1}+\lambda_{\max }\left(\mathbf{A}_{P U}\right)
$$

\section{SRB WITH THE EAVESDROPPER'S CSI}

We assume that eavesdropper's CSI can be estimate at the two-way relay and the source1 and the source2, which is valid when the eavesdropper is active [10]. When the eavesdropper's CSI is available, we all works are to maximize (30)

$$
\left(\begin{array}{l}
\frac{1}{2} \log _{2}\left(1+\frac{\mathbf{w}^{H}\left(\mathbf{H}_{S 2 f}^{H} \mathbf{H}_{S 2 f}^{H} \otimes \mathbf{H}_{S 1 b}^{T} \mathbf{H}_{S 1 b}^{*}\right) \mathbf{w}}{\mathbf{w}^{H}\left(\sigma^{2} \mathbf{I} \otimes \mathbf{H}_{S 1 b}^{T} \mathbf{H}_{S 1 b}^{*}\right) \mathbf{w}+\sigma^{2}}\right) \\
+\frac{1}{2} \log _{2}\left(1+\frac{\mathbf{w}^{H}\left(\mathbf{H}_{S 1 f}^{H} \mathbf{H}_{S 1 f}^{H} \otimes \mathbf{H}_{S 2 b}^{T} \mathbf{H}_{S 2 b}^{*}\right) \mathbf{w}}{\mathbf{w}^{H}\left(\sigma^{2} \mathbf{I} \otimes \mathbf{H}_{S 2 b}^{T} \mathbf{H}_{S 2 b}^{*}\right) \mathbf{w}+\sigma^{2}}\right) \\
-\frac{1}{2} \log _{2}\left(1+\frac{\mathbf{w}^{H} \mathbf{Q}_{e} \mathbf{w}+\alpha}{\left(1+\mathbf{w}^{H} \mathbf{R}_{c c} \mathbf{w}\right) \sigma^{4}}\right)
\end{array}\right)
$$

$$
\begin{aligned}
& \text { s.t. } \mathrm{E}_{1}+\mathrm{E}_{2} \geq \mathrm{Q}_{\text {th }} \\
& \mathrm{E}_{p 1}+\mathrm{E}_{p 2} \leq I_{t h} \\
& \mathbf{w}^{H}\left(\mathbf{Y}_{y}^{T} \otimes \mathbf{I}\right) \mathbf{w} \leq P_{t h}
\end{aligned}
$$

According to Lemma 1 in [11], the optimal beamforming vector $\mathbf{W}$ in (30) is

(1) when $\mathrm{P}_{\text {th }} \operatorname{tr}\left(\mathbf{p}_{r}^{*} \mathbf{p}^{\mathrm{T}}\right) \leq I_{t h}-E_{p 1}$

$$
\mathbf{w}^{H} \mathbf{A}_{\text {Relay }} \mathbf{w}=P_{\text {th }}
$$

where $\mathbf{A}_{\text {Relay }}=\mathbf{Y}_{y}^{T} \otimes \mathbf{I}$.

(2) when $\mathrm{P}_{\mathrm{th}} \operatorname{tr}\left(\mathbf{p}_{r}^{*} \mathbf{p}_{r}^{T}\right)>I_{t h}-E_{p 1}$

$$
\mathbf{w}^{H} \mathbf{A}_{\text {Relay }} \mathbf{w}=\left(I_{t h}-E_{p 1}\right) \operatorname{tr}\left(\mathbf{p}_{r}^{*} \mathbf{p}_{r}^{T}\right)^{-1}
$$

Fixing $P_{2},(30)$ is monotonically increase or decrease with respect to $P_{1}[11]$. If (1) is established, we set $\mathbf{M}_{1}=\mathbf{A}_{\text {Relay }} \mathrm{P}_{\text {th }}^{-1}$. Otherwise, $\mathbf{M}_{1}=\mathbf{A}_{\text {Relay }}\left(\mathrm{I}_{\text {th }}-\mathrm{E}_{\mathrm{pl}}\right)^{-1} \operatorname{tr}\left(\mathbf{p}_{r}^{*} \mathbf{p}_{r}^{T}\right)$.

(33) is established

$$
\mathbf{w}^{\mathrm{H}} \mathbf{M}_{1} \mathbf{w}=1
$$

We substitute (33) into problem (30), since $\frac{1}{2} \log _{2}$ is monotonically increase function and omit $\frac{1}{2} \log _{2}$ can simplification computation. we omit $\frac{1}{2} \log _{2}$ and get

$$
\begin{gathered}
\frac{\mathbf{w}^{H} \mathbf{A}_{11} \mathbf{w}}{\mathbf{w}^{H} \mathbf{A}_{12} \mathbf{w}} \cdot \frac{\mathbf{w}^{H} \mathbf{B}_{11} \mathbf{w}}{\mathbf{w}^{H} \mathbf{B}_{12} \mathbf{w}} \cdot \frac{\mathbf{w}^{H} \mathbf{C}_{11} \mathbf{w}}{\mathbf{w}^{H} \mathbf{C}_{12} \mathbf{w}} \\
\mathbf{w}^{H} \mathbf{D}_{11} \mathbf{w}-\mathbf{w}^{H} \mathbf{D}_{12} \mathbf{w} \leq 0 \\
\mathbf{w}^{H} \mathbf{E}_{11} \mathbf{w}-\mathbf{w}^{H} \mathbf{E}_{12} \mathbf{w} \leq 0
\end{gathered}
$$

where

$$
\begin{aligned}
& \mathbf{A}_{11}=\left(\begin{array}{l}
\mathrm{P}_{2}\left(\mathbf{H}_{\mathrm{S} 2 f}^{H} \mathbf{H}_{\mathrm{S} 2 f} \otimes \mathbf{H}_{\mathrm{S} 1 b}^{T} \mathbf{H}_{\mathrm{S} 1 b}^{*}\right)+\left(\sigma^{2} \mathbf{I} \otimes \mathbf{H}_{\mathrm{S} 1 b}^{T} \mathbf{H}_{\mathrm{S} 1 b}^{*}\right) \\
+\sigma^{2} \mathbf{M}_{1}
\end{array}\right) \\
& \mathbf{A}_{12}=\left(\sigma^{2} \mathbf{I} \otimes \mathbf{H}_{\mathrm{S} 1 b}^{T} \mathbf{H}_{\mathrm{S} 1 b}^{*}\right)+\sigma^{2} \mathbf{M}_{1} \\
& \mathbf{B}_{12}=\left(\sigma^{2} \mathbf{I} \otimes \mathbf{H}_{\mathrm{S} 2 b}^{T} \mathbf{H}_{\mathrm{S} 2 b}^{*}\right)+\sigma^{2} \mathbf{M}_{1}
\end{aligned}
$$




$$
\begin{aligned}
& \mathbf{C}_{11}=\left(\mathbf{R}_{c c}+\mathbf{M}_{1}\right) \sigma^{4}, \mathbf{C}_{12}=\mathbf{Q}_{e}+\alpha \mathbf{M}_{1}, \\
& \mathbf{B}_{11}=\left(\begin{array}{l}
\mathbf{P}_{1}\left(\mathbf{H}_{\mathrm{S} 1 f}^{H} \mathbf{H}_{\mathrm{S} 1 f} \otimes \mathbf{H}_{\mathrm{S} 2 b}^{T} \mathbf{H}_{\mathrm{S} 2 b}^{*}\right) \\
+\left(\sigma^{2} \mathbf{I} \otimes \mathbf{H}_{\mathrm{S} 2 b}^{T} \mathbf{H}_{\mathrm{S} 2 b}^{*}\right)+\sigma^{2} \mathbf{M}_{1}
\end{array}\right) \\
& \mathbf{D}_{11}=\left(\mathbf{Q}_{t h}-E_{1}\right) \mathbf{M}_{1}, \mathbf{D}_{12}=\mathbf{A}_{\mathrm{EH} 2}, \\
& \mathbf{E}_{11}=\mathbf{A}_{\mathrm{PU}}+\mathrm{E}_{p 1} \mathbf{M}_{1}, \mathbf{E}_{12}=\mathbf{I}_{t h} \mathbf{M}_{1} .
\end{aligned}
$$

\section{A. 2D Search Solution}

In this section, we employ 2D Search and to derive an upper bound for (34). First, we introduce two slack variables $\operatorname{var}_{1}$ and $\operatorname{var}_{2}$.

$$
\operatorname{var}_{1} \leq \frac{\mathbf{w}^{H} \mathbf{A}_{11} \mathbf{w}}{\mathbf{w}^{H} \mathbf{A}_{12} \mathbf{w}}, \operatorname{var}_{1} \leq \frac{\mathbf{w}^{H} \mathbf{B}_{11} \mathbf{w}}{\mathbf{w}^{H} \mathbf{B}_{12} \mathbf{w}}
$$

Problem (34) turn into (36)

$$
\begin{aligned}
& \max _{\mathbf{w}, \mathrm{var}_{1}, \mathrm{var}_{2}}\left(\operatorname{var}_{1} \cdot \operatorname{var}_{2} \cdot \frac{\mathbf{w}^{H} \mathbf{C}_{11} \mathbf{w}}{\mathbf{w}^{H} \mathbf{C}_{12} \mathbf{w}}\right) \\
& \text { s.t. } \mathbf{w}^{H}\left(\mathbf{A}_{11}-\operatorname{var}_{1} \mathbf{A}_{12}\right) \mathbf{w} \geq 0 \\
& \mathbf{w}^{H}\left(\mathbf{B}_{11}-\operatorname{var}_{2} \mathbf{B}_{12}\right) \mathbf{w} \geq 0 \\
& \mathbf{w}^{H}\left(\mathbf{D}_{11}-\mathbf{D}_{12}\right) \mathbf{w} \geq 0 \\
& \mathbf{w}^{H}\left(\mathbf{E}_{11}-\mathbf{E}_{12}\right) \mathbf{w} \geq 0
\end{aligned}
$$

By employing rank-one relaxation and using the equality $\mathbf{Q}=\mathbf{W w}^{H}$. (36) can be expressed as

$$
\begin{gathered}
\max _{\mathbf{Q} \geq 0, \text { var }_{1}, \text { var }_{2}}\left(\operatorname{var}_{1} \cdot \operatorname{var}_{2} \cdot \frac{\operatorname{tr}\left(\mathbf{C}_{11} \mathbf{Q}\right)}{\operatorname{tr}\left(\mathbf{C}_{12} \mathbf{Q}\right)}\right) \\
\text { s.t } \operatorname{tr}\left(\left(\mathbf{A}_{11}-\operatorname{var}_{1} \mathbf{A}_{12}\right) \mathbf{Q}\right) \geq 0 \\
\operatorname{tr}\left(\left(\mathbf{B}_{11}-\operatorname{var}_{2} \mathbf{B}_{12}\right) \mathbf{Q}\right) \geq 0 \\
\operatorname{tr}\left(\left(\mathbf{D}_{11}-\mathbf{D}_{12}\right) \mathbf{Q}\right) \leq 0 \\
\operatorname{tr}\left(\left(\mathbf{E}_{11}-\mathbf{E}_{12}\right) \mathbf{Q}\right) \leq 0
\end{gathered}
$$

If problem (37) has an rank-one $\mathbf{Q}$, (37) is equivalent to problem (36). Given $\operatorname{var}_{1}$ and $\operatorname{var}_{2}$, problem (37) is a linear fractional programming, which can be solved by employing Charnes-Cooper transformation.

$$
\max _{\mathbf{Q} \geq 0}\left(\operatorname{var}_{1} \cdot \operatorname{var}_{2} \cdot \operatorname{tr}\left(\mathbf{C}_{11} \mathbf{Q}\right)\right)
$$

$$
\text { s.t. } \operatorname{tr}\left(\mathbf{C}_{12} \mathbf{Q}\right)=1
$$

(37a)-(37d)

where (38) is an SDP problem. We can solve the SDP utilize the interior-point method. (35) are generalized Rayleigh quotients. Duo to the properties of generalized Rayleigh quotient, we obtain

$$
\begin{aligned}
& \lambda_{\text {max }}\left(\mathbf{A}_{11} \mathbf{A}_{12}^{-1}\right) \leq \operatorname{var}_{1} \leq \lambda_{\text {min }}\left(\mathbf{A}_{11} \mathbf{A}_{12}^{-1}\right) \\
& \lambda_{\text {max }}\left(\mathbf{B}_{11} \mathbf{B}_{12}^{-1}\right) \leq \operatorname{var}_{2} \leq \lambda_{\text {min }}\left(\mathbf{B}_{11} \mathbf{B}_{12}^{-1}\right)
\end{aligned}
$$

If the optimal solution to (38) found by programming is not rank-one, we can utilize the Gaussian randomization method to find a sub-optimal solution [13].

\section{B. SPCA Based Iteration Scheme}

In this section, we develop a lower complexity method to find a local optimal solution. According to [11], the conclusion has shown that the SPCA based locally optimal solution performs close to upper bound. To solve (34), we transform it into an equivalent difference convex problem (DCP). It can be expressed as

$$
\begin{gathered}
\max _{\mathbf{w}}\left(\begin{array}{c}
\ln \left(\mathbf{w}^{H} \mathbf{A}_{11} \mathbf{w}\right)+\ln \left(\mathbf{w}^{H} \mathbf{B}_{11} \mathbf{w}\right)+\ln \left(\mathbf{w}^{H} \mathbf{C}_{11} \mathbf{w}\right) \\
-\ln \left(\mathbf{w}^{H} \mathbf{A}_{12} \mathbf{w}\right)-\ln \left(\mathbf{w}^{H} \mathbf{B}_{12} \mathbf{w}\right)-\ln \left(\mathbf{w}^{H} \mathbf{C}_{12} \mathbf{w}\right)
\end{array}\right) \\
\text { s.t. (34a)-(34b) }
\end{gathered}
$$

We utilize the equality $\mathbf{Q}=\mathbf{W w}^{H}$ and transform (41) into

$$
\min _{\mathbf{Q} \geq 0}\left(\begin{array}{l}
-\ln \left(\operatorname{tr}\left(\mathbf{A}_{11} \mathbf{Q}\right)\right)-\ln \left(\operatorname{tr}\left(\mathbf{B}_{11} \mathbf{Q}\right)\right)-\ln \left(\operatorname{tr}\left(\mathbf{C}_{11} \mathbf{Q}\right)\right) \\
+\ln \left(\operatorname{tr}\left(\mathbf{A}_{12} \mathbf{Q}\right)\right)+\ln \left(\operatorname{tr}\left(\mathbf{B}_{12} \mathbf{Q}\right)\right)+\ln \left(\operatorname{tr}\left(\mathbf{C}_{12} \mathbf{Q}\right)\right)
\end{array}\right)
$$

s.t. $\operatorname{Rank}(\mathbf{Q})=1$

(37c)-(37d)

where $\operatorname{Rank}(\mathbf{Q})=1$ with $\mathbf{Q} \geq 0$ can be equivalent to (43) [12]

$$
\begin{aligned}
& \mathbf{Q} \geq 0 \\
& \operatorname{tr}(\mathbf{Q})-\lambda_{\text {max }}(\mathbf{Q}) \leq 0
\end{aligned}
$$

We employ (43) turn (42) into (44)

$$
\begin{aligned}
& \qquad \min _{\mathbf{Q} \geq 0}\left(\begin{array}{l}
\ln \left(\operatorname{tr}\left(\mathbf{A}_{12} \mathbf{Q}\right)\right)+\ln \left(\operatorname{tr}\left(\mathbf{B}_{12} \mathbf{Q}\right)\right)+\ln \left(\operatorname{tr}\left(\mathbf{C}_{12} \mathbf{Q}\right)\right) \\
-\ln \left(\operatorname{tr}\left(\mathbf{A}_{11} \mathbf{Q}\right)\right)-\ln \left(\operatorname{tr}\left(\mathbf{B}_{11} \mathbf{Q}\right)\right)-\ln \left(\operatorname{tr}\left(\mathbf{C}_{11} \mathbf{Q}\right)\right)
\end{array}\right) \\
& \text { s.t. } \operatorname{tr}(\mathbf{Q})-\lambda_{\max }(\mathbf{Q}) \leq 0
\end{aligned}
$$


$(37 \mathrm{c})-(37 \mathrm{~d})$

We utilize the exact penalty method [13] to solve (44) and rewrite it

$$
\min _{\mathbf{Q} \geq 0}\left(\begin{array}{l}
\ln \left(\operatorname{tr}\left(\mathbf{A}_{12} \mathbf{Q}\right)\right)+\ln \left(\operatorname{tr}\left(\mathbf{B}_{12} \mathbf{Q}\right)\right)+\ln \left(\operatorname{tr}\left(\mathbf{C}_{12} \mathbf{Q}\right)\right) \\
-\ln \left(\operatorname{tr}\left(\mathbf{A}_{11} \mathbf{Q}\right)\right)-\ln \left(\operatorname{tr}\left(\mathbf{B}_{11} \mathbf{Q}\right)\right)-\ln \left(\operatorname{tr}\left(\mathbf{C}_{11} \mathbf{Q}\right)\right) \\
+\varepsilon\left(\operatorname{tr}(\mathbf{Q})-\lambda_{\text {max }}(\mathbf{Q})\right)
\end{array}\right)
$$

$$
\text { s.t. (37c)-(37d) }
$$

where $\varepsilon$ is an enough large penalty factor. For problem (45) is equivalent to problem (42) when $0 \prec \varepsilon \prec+\infty$ [11]. We use the result of [14]. If we replace the non-convex terms with their convex upper bounds and iterative solve the resulting problem by judiciously updating the variables until convergence. we can obtain a local optimum of (45). We employ this method to replace (46) and (47)

$$
\begin{gathered}
-\lambda_{\text {max }}(\mathbf{Q}) \\
\ln \left(\operatorname{tr}\left(\mathbf{A}_{12} \mathbf{Q}\right)\right)+\ln \left(\operatorname{tr}\left(\mathbf{B}_{12} \mathbf{Q}\right)\right)+\ln \left(\operatorname{tr}\left(\mathbf{C}_{12} \mathbf{Q}\right)\right)
\end{gathered}
$$

We assume that $\mathbf{Q}_{\mathbf{q}}$ is an available point to problem (45), using the property of concave functions, we have

$$
\begin{aligned}
& \ln (\operatorname{tr}(\mathbf{A} \mathbf{Q})) \leq \ln \left(\operatorname{tr}\left(\mathbf{A} \mathbf{Q}_{q}\right)\right)+\frac{\operatorname{tr}\left(\mathbf{A}\left(\mathbf{Q}-\mathbf{Q}_{q}\right)\right)}{\operatorname{tr}\left(\mathbf{A} \mathbf{Q}_{q}\right)} \\
& -\lambda_{\max }(\mathbf{Q}) \leq-\lambda_{\text {max }}\left(\mathbf{Q}_{q}\right)-\operatorname{tr}\left(\mathbf{q q}{ }^{H}\left(\mathbf{Q}-\mathbf{Q}_{q}\right)\right)
\end{aligned}
$$

where $\mathbf{q}$ is the unit-norm eigenvector corresponding to the maximal eigenvalue $\lambda_{\text {max }}\left(\mathbf{Q}_{q}\right)$.

Since the right-hand sides of (48) and (49) are linear, they are convex upper bounds of the left-hand sides of (48) and (49), respectively.

$$
\min _{\mathbf{Q} \geq 0}\left(\begin{array}{l}
\frac{\operatorname{tr}\left(\mathbf{A}_{12} \mathbf{Q}\right)}{\operatorname{tr}\left(\mathbf{A}_{12} \mathbf{Q}^{(n)}\right)}+\frac{\operatorname{tr}\left(\mathbf{B}_{12} \mathbf{Q}\right)}{\operatorname{tr}\left(\mathbf{B}_{12} \mathbf{Q}^{(n)}\right)}+\frac{\operatorname{tr}\left(\mathbf{C}_{12} \mathbf{Q}\right)}{\operatorname{tr}\left(\mathbf{C}_{12} \mathbf{Q}^{(n)}\right)} \\
-\ln \left(\operatorname{tr}\left(\mathbf{A}_{11} \mathbf{Q}\right)\right)-\ln \left(\operatorname{tr}\left(\mathbf{B}_{11} \mathbf{Q}\right)\right) \\
-\ln \left(\operatorname{tr}\left(\mathbf{C}_{11} \mathbf{Q}\right)\right)+\varepsilon\left(\operatorname{tr}(\mathbf{Q})-\operatorname{tr}\left(\mathbf{q}^{(n)} \mathbf{q}^{(n) H} \mathbf{Q}\right)\right)
\end{array}\right)
$$

$$
\text { s.t. }(37 c)-(37 d)
$$

In (50), $\mathbf{Q}^{(n)}$ is optimal solution in the $n$th iteration, $\mathbf{q}^{(n)}$ is the unit-norm eigenvector corresponding to the maximal eigenvalue $\lambda_{\max }\left(\mathbf{Q}^{(n)}\right) \cdot \mathbf{Q}^{(0)}$ is chosen by using $\mathbf{U} \mathbf{x}{ }^{H} \mathbf{U}^{H}$, where $\mathbf{U}$ are consists of the eigenvectors of matrix $\mathbf{D}_{11}-\mathbf{D}_{12}$ which are associated with the eigenvalues being no greater than zero. $\mathbf{X}$ is a randomly generated vector. We solve the convex optimization problem base on SPCA iterative algorithm until convergence.

\section{COMPLEXITY ANALYSIS}

From [11], the computational complexity of 2D search scheme and SPCA based iteration scheme are

$$
\begin{gathered}
o\left(\mathrm{~L}_{1} \mathrm{~L}_{2} \sqrt{\mathrm{N}^{2}+5}\left(5 \mathrm{~N}^{6}+25 \mathrm{~N}^{4}+215\right)\right) \\
o\left(\mathrm{~L} \sqrt{\mathrm{N}^{2}+2}\left(\mathrm{~N}^{6}+\mathrm{N}^{4}+3\right)\right)
\end{gathered}
$$

where The $L_{1}$ and $L_{2}$ is the number of $2 \mathrm{D}$ search scheme. $\mathrm{L}$ is the number of iterations of the SPCA based iteration scheme.

\section{SIMULATION RESULTS}

In this section, we will present the simulation results. First we assume that in the two-way relay network all the entries in the channel response vectors are i. i. d. Complex

Gaussian random variables with zero mean and unit variance. To solve the SDPs, we use the Matlab-based CVX optimization software. In all simulations, the transmit power of two sources are $\mathrm{P}_{1}=\mathrm{P}_{2}=5 d B$.

In Fig. 2, we present the average sum rate comparison of different secure relay beamforming schemes for various $\mathrm{P}_{t h} / \sigma^{2}$ constraint. In the legend, 2D search scheme is denoted as $2 \mathrm{D}$, and the SPCA based iteration scheme is denoted as SPCA. The EH constraint is $\mathrm{Q}_{\mathrm{th}}=0.5 Q_{\max }$ and the PU interference threshold is $\mathrm{I}_{t h}=\mathrm{I}_{\text {thmax }}$. The number of antennas at relay is $\mathrm{N}=4$. In this paper, $\varepsilon=0.01$. From Fig. 2 , with the increase of $\mathrm{P}_{t h} / \sigma^{2}$, the average capacity increase. When $\mathrm{P}_{t h} / \sigma^{2}$ reaches to $20 \mathrm{~dB}$, the average capacity stay the same. It is observed from Fig. 2 that the SPCA scheme performance is very close to 2D scheme.

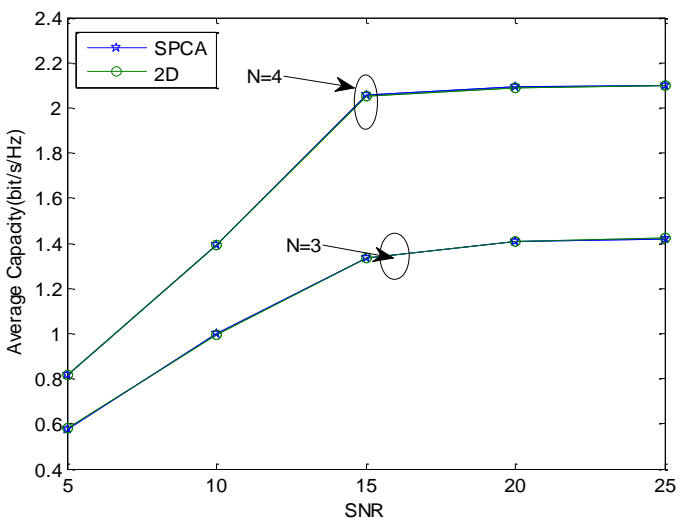

Figure 2. Average secrecy sum capacity versus SNR 


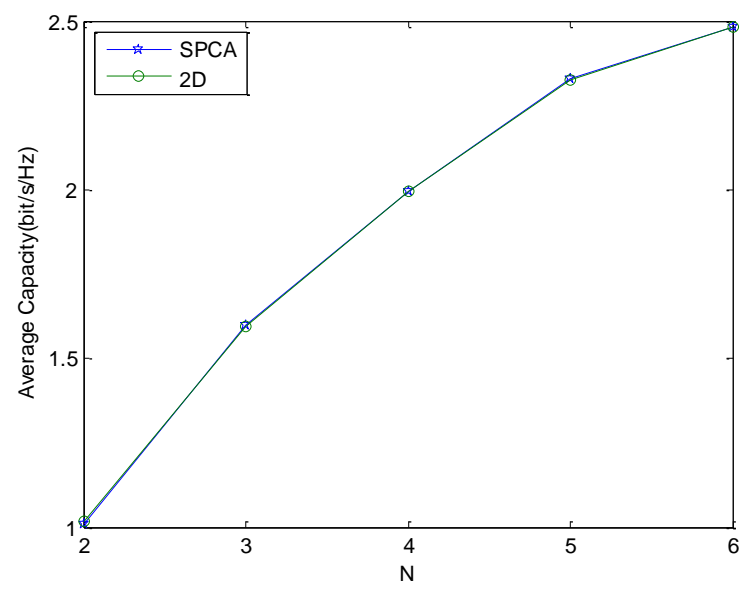

Figure 3. Average secrecy sum capacity versus N

In Fig. 3, we present the average sum rate comparison of different schemes for different number of antennas at relay where the transmit power to noise power ratio of relay is $\mathrm{P}_{t h} / \sigma^{2}=20 \mathrm{~dB}$, and the $\mathrm{EH}$ constraints is $\mathrm{Q}_{\mathrm{th}}=0.5 Q_{\max }$ and the $\mathrm{PU}$ interference threshold is $\mathrm{I}_{\text {th }}=\mathrm{I}_{\text {thmax }}$. It is observed from Fig. 3 that with the increase of $\mathrm{N}$, the average sum rates of our proposed schemes increase.

\section{CONCLUSIONS}

In this paper, we propose two schemes, 2D search scheme and SPCA based iteration scheme, to solve the problem of secure relay beamforming in the nonregenerative two-way relay cognitive radio networks when the eavesdropper's CSI is available. Simulation results have shown that SPCA based iteration scheme performs almost close to 2D search schemes, and has lower computation complexity.

\section{ACKNOWLEDGEMENT}

This work is supported by National natural science foundation of China (61403089), Guangdong science \& technology project (2013B010402018, 2013B020200016, 2016A0101032), Guangzhou science \& technology found
(2014J4100142, 2014J4100233), Guangzhou education bureau science and technology project (2012A082, 1201421329, 1201630400).

\section{REFERENCES}

[1] S. Hakin "Cognitive Radio: brain-empowered wireless communications," IEEE J.Sel. Areas Commun, vol. 23, no. 2, pp 201-220, Feb 2005.

[2] A. Wyner "The wiretap channel," Bell. Syst Tech. J, vol. 54, no. 8, pp. 1355-1387, Jan 1975

[3] I. Csiszar and J.Korner "Broadcast channel with confidential," IEEE Trans. Infor. Theory, vol. IT-24, no.3, pp. 339-348, May 1978.

[4] L. Dong, Z. Han, A. Petropulu and H. V. Poor," Secure wireless communications via cooperation," Proc. 46th Annual Allerton Conf. Commun, Control, and Computing, Monticello, IL, Sept 2008.

[5] G. Huang, D. Tang, "Wireless Information and Power Transfer in Two-Way OFDM," IEEE Commun. Lett., vol. 20, no. 8, pp. 15631566, Aug. 2016

[6] A. Khabbazibasmenj and F. Roemer, "Sum-rate maximization in two -way AF," IEEE trans. Signal Process., vol. 60, no. 10, Oct. 2012.

[7] H.-M. Wang, Q. Yin, and X.-G. Xia, "Distributed beamforming for physical-layer security of two-way relay networks," IEEE Trans. Signal Process., vol. 60, no. 7, pp. 3532-3545, Jul. 2012.

[8] R. Zhang and C. K. Ho, "MIMO broadcasting for simultaneous wireless information and power transfer," IEEE Trans. Wireless Commun., vol. 12, no. 5, pp. 1989-2001, May 2013.

[9] C. Xing, N. Wang, J. Ni, Z. Fei, and J. Kuang, "MIMO beamforming designs with partial CSI under energy harvesting constraints," IEEE Signal Process. Lett., vol. 20, no. 4, pp. 363-366, Apr. 2013.

[10] P. K. Gopala, L. Lai, and H. El Gamal, "On the secrecy capacity of fading channels," IEEE Trans. Inf. Theory, vol. 54, no. 10, pp. 46864698, Oct. 2008.

[11] Q. Li, Q. Zhang, and J. Qin, "Secure Relay Beam-forming for SWIPT in Non-Regenerative,"IEEE Trans. Wireless Commun., Veh. Technol., vol. 65 , no. 11, pp. 9006 - 9019, Nov. 2016

[12] S. Boyd and L. Vandenberghe, Convex Optimization. Cambridge, U.K.:Cambridge Univ. Press, 2004.

[13] M. A. M.Sadr and M. A. Attari "secure robust relay beamforming : a convex conic approximation approach" IET comun. ,vol. 10, Iss. 10, pp.1138-1150,2016.

[14] A. Beck, A. Ben-Tal, and L. Tetruashvili, "A sequential parametric convex approximation method with applications to nonconvex truss topology design problems," J. Global Optim., vol. 47, no. 1, pp. 2951,2010 .

[15] D. Tang, D. Jiang, G. Huang, M. Xia, "Energy states aided relay selection and optimal power allocation for cognitive relaying networks," IET Commun., accepted. 